\title{
The Doubly Periodic Scherk-Costa Surfaces
}

\author{
Kelly Roberta Mazzutti Lübeck ${ }^{1} \&$ Valério Ramos Batista ${ }^{2}$ \\ ${ }^{1}$ CECE, Unioeste, Foz do Iguaçu, Brazil \\ ${ }^{2}$ CMCC, UFABC, St André, Brazil \\ Correspondence: Kelly Roberta Mazzutti Lübeck, CECE, Unioeste, Foz do Iguaçu, PR 85870-650, Brazil. Tel: \\ 55-45-3576-8100. E-mail: klubeck@ unioeste.br
}

\author{
Received: December 11, 2013 Accepted: February 20, 2014 Online Published: April 18, 2014 \\ doi:10.5539/jmr.v6n2p77 URL: http://dx.doi.org/10.5539/jmr.v6n2p77
}

\begin{abstract}
We present a new family of embedded doubly periodic minimal surfaces, of which the symmetry group does not coincide with any other example known before.
\end{abstract}

Keywords: embedded, doubly periodic, minimal surfaces

\section{Introduction}

Any complete embedded minimal surface in $\mathbb{R}^{3}$ belongs to one of four different classes: non-periodic, singly, doubly or triply periodic. Explicit examples are only known for finite topology (after the quotient by translations) and for proper embeddedness. In this case, the triply periodic class has always been the richest one, and this fact counts to a great extent on the Conjugate Plateau Construction. This is a very powerful method explained in Sections 4 and 6 of Karcher and Polthier (1996), but it requires a fundamental conjugate frame that projects onto a convex plane region bounded by a monotone curve. In Ramos Batista (2003), one obtains explicit examples that do not fulfil this requirement. Hence, the Conjugate Plateau Construction is not always applicable, or even extendable to infinite frames (See Jenkins \& Serrin, 1966).

The less numerous in explicit examples is the doubly periodic class. This is probably due to the fact, proved in Meeks and Rosenberg (1989), that all such surfaces must have only Scherk-type ends. Much more flexible is the singly periodic class, which allows three different kinds of ends: planar, Scherk or helicoidal. Regarding the nonperiodic class, if the surface has finite total curvature, only planar or catenoidal ends come out. Otherwise, it must have a helicoidal end (See Hoffman, Karcher, \& Wei, 1993; Hoffman, Wolf, \& Weber, 2009; Meeks \& Rosenberg, 2005).

In the $20^{\text {th }}$ century, the explicit examples were obtained thanks to their high order symmetry groups, a resource already used up nowadays. Therefore, potentially new examples normally lack in symmetries, which makes it so hard to prove their existence. One might opt for keeping a high order symmetry group with an increase in the genus, but this leads to the same hurdle, namely too many period problems.

Several non-explicit examples were obtained by modern constructions, which do close many periods at once. For instance, in Kapouleas (1997) the author desingularizes a finite set of co-axial intersecting catenoids and planes, and obtains minimal surfaces with high genera that, however, cannot be determined. In Traizet (1996) the author uses heavy Functional Analysis to desingularize vertical intersecting planes into singly periodic surfaces with Scherk ends, however without getting Weierstraß Data. In Traizet (2002a, 2002b) he now uses an implicit function argument to obtain his surfaces, but this requires hard computation of partial derivatives of elliptic integrals. Teichmüller Theory is applied in Weber (2000) for singly periodic constructions, but this theory is far from being trivial. Finally, singly and doubly periodic examples are obtained in Hauswirth, Morabito, and Rodíguez (2009) by a cut-and-glue procedure, again with heavy Functional Analysis and without Weierstraß Data.

For at most three period problems, however, it is still feasible to handle them with much less computations, providing one applies some adequate methods (See Baginski \& Ramos Batista, 2011; Lübeck, 2007; Lübeck \& Ramos Batista, 2009; Martín \& Ramos Batista, 2006). By the way, in this paper we solve three periods at once by applying the limit-method described both in Lübeck (2007), Lübeck and Ramos Batista (2009). 
However, what is the purpose of constructing a new minimal surface? The following reason motivates this present work. Minimal surfaces model many structures, like crystals and co-polymers, but several symmetry groups are not yet represented by any of them (See Hart, 2007; Lord \& Mackay, 2003). As explained above, the doubly periodic class still lacks in examples, even after very rich works like Hoffman, Karcher, and Wei (1993), Karcher (1988), Pérez, Rodríguez, and Traizet (2005), Rossman, Thayer, and Wohlgemuth (2000), and Wei (1992). A survey of such works indicates what symmetry groups already have a representative. The purpose of this paper is then to introduce a new family of embedded doubly periodic minimal surfaces, of which the symmetry group does not coincide with any other example known before. For the converse, there are symmetry groups that admit more than one representative, even restricted to a certain conformal type (See Ramos Batista, 2004). Although not embedded, these examples easily hint at embedded ones.

Our surfaces are inspired in the examples called $\mathrm{L}_{\mathrm{b}}$ in Ramos Batista (2005, p. 482). By taking the picture of $\mathrm{L}_{\mathrm{b}}$ on that page, if one replaces the catenoidal ends by curves of reflectional symmetry parallel to $O x_{1} x_{2}$, the resultant surface will then come out as in Figure 1. Its symmetry group is $D_{2 d}$ or $\overline{4} 2 m$ in Schönflies or in simplified Hermann-Maugin notation, respectively. The same procedure for $\mathrm{C}_{\mathrm{b}}$ from Ramos Batista (2005, p. 483) could also result in a new doubly periodic example. However, it would then have the same symmetry group as $M_{\pi / 4, \pi / 2,0}$ from Pérez, Rodríguez and Traizet (2005). Of course, they would differ in genus, but one still might go round it by adding handles to the latter, a widely applicable technique. That is why our paper is totally devoted to the example in Figure 1.

We formally state our result in the following theorem:

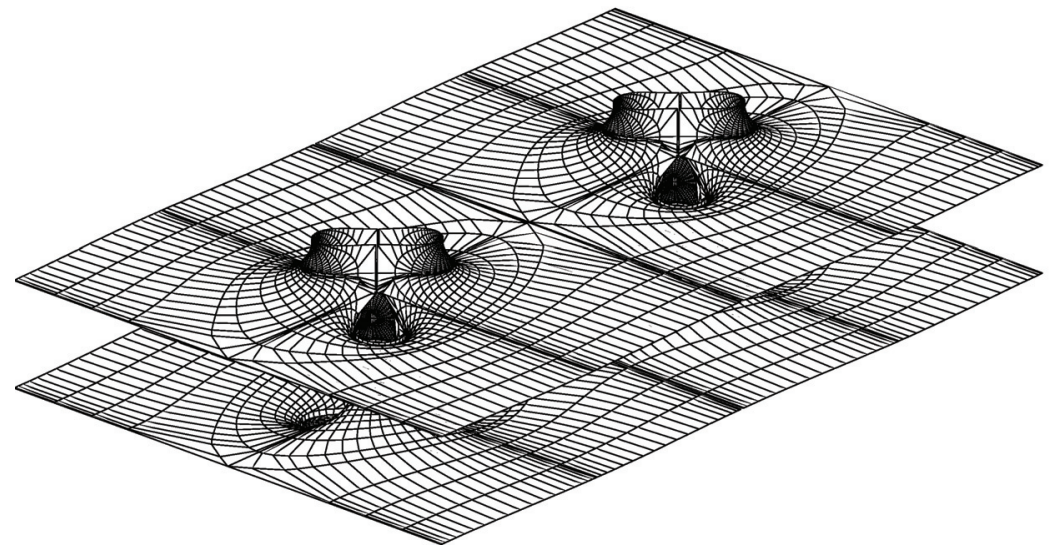

Figure 1. A doubly periodic Scherk-Costa surface

Theorem 1 There exists a one-parameter family of complete embedded doubly periodic minimal surfaces in $\mathbb{R}^{3}$ such that, for each member of this family the following holds:

(a) The quotient by its translation group has genus three. Up to vertical translation and re-scaling, this quotient is invariant by a reflection in either plane $x_{3}= \pm 1$, which turns out to be the same reflection. Further quotient by this reflection gives a smaller surface called fundamental piece.

(b) The doubly periodic surface is generated by the fundamental piece, which is a surface with boundary in $\mathbb{R}^{3}$. The fundamental piece has two Scherk-ends (modulo translation), and a symmetry group generated by $180^{\circ}$-rotations around a straight line and $180^{\circ}$-rotations around a straight segment. The segment crosses the line orthogonally and both determine a plane $\Pi$.

(c) The boundary of the fundamental piece consists of two parallel lines in $\Pi$ and two planar closed curves of reflectional symmetry. The curves are parallel to but not contained in $\Pi$, and one is the image of the other under the symmetries of the fundamental piece. By successive $180^{\circ}$-rotations around the lines of the boundary and reflections in the closed curves, one generates the doubly periodic surface.

The crucial example for the proof of Theorem 1 is the Callahan-Hoffman-Meeks' surface of genus 3, described in Callahan, Hoffman, and Meeks (1989). In this work the authors construct singly periodic examples, of which the quotient by translation has two planar ends and genus $2 k+1$, where $k$ ranges in $\mathbb{N}^{*}$. Our surfaces build a family with boundary that limit to their example $k=1$. Figures 1 and 7 may be compared to understand this fact. 
The present work uses part of our doctoral theses Lübeck (2007) and Ramos Batista (2000), supported by CAPES (Coordenação de Aperfeiçoamento de Pessoal de Nível Superior) and DAAD (Deutscher Akademischer Austausch Dienst), respectively. Professor Hermann Karcher, from the University of Bonn in Germany, was adviser of the second author, who thanks him for his dedication, which greatly helped in the realisation of this work. The second author was advisor of the first.

\section{Preliminaries}

In this section we state some well known theorems on minimal surfaces. For details, we refer the reader to Karcher (1989), López and Martín (1999), Nitsche (1989), and Osserman (1986). In this paper all surfaces are supposed to be regular.

Theorem 2 (Weierstraß representation) Let $R$ be a Riemann surface, $g$ and $d h$ meromorphic function and 1 differential form on $R$, respectively, such that the zeros of $d$ h coincide with the poles and zeros of $g$. Consider the (possibly multi-valued) function $X: R \rightarrow \mathbb{R}^{3}$ given by

$$
X(p):=\operatorname{Re} \int^{p}\left(\phi_{1}, \phi_{2}, \phi_{3}\right), \text { where }\left(\phi_{1}, \phi_{2}, \phi_{3}\right):=\frac{1}{2}\left(g^{-1}-g, i g^{-1}+i g, 2\right) d h .
$$

Then $X$ is a conformal minimal immersion. Conversely, every conformal minimal immersion $X: R \rightarrow \mathbb{R}^{3}$ can be expressed as above for some meromorphic function $g$ and 1-form $\mathrm{dh}$.

Definition 1 The pair $(g, d h)$ is the Weierstra $\beta$ data and $\phi_{1}, \phi_{2}, \phi_{3}$ are the Weierstra $\beta$ forms on $R$ of the minimal immersion $X: R \rightarrow X(R)=S \subset \mathbb{R}^{3}$.

Definition 2 A complete, orientable minimal surface $S$ is algebraic if it admits a Weierstraß representation such that $R=\bar{R} \backslash\left\{p_{1}, \ldots, p_{r}\right\}$, were $\bar{R}$ is compact, and both $g$ and $d h$ extend meromorphically to $\bar{R}$.

Definition 3 An end of $S$ is the image of a punctured neighbourhood $V_{p}$ of a point $p \in\left\{p_{1}, \ldots, p_{r}\right\}$ such that $\left(\left\{p_{1}, \ldots, p_{r}\right\} \backslash\{p\}\right) \cap \bar{V}_{p}=\emptyset$. The end is embedded if this image is embedded for a sufficiently small neighbourhood of $p$.

Theorem 3 Let $S$ be a complete minimal surface in $\mathbb{R}^{3}$. Then $S$ is algebraic if and only if it can be obtained from a piece $\tilde{S}$ of finite total curvature by applying a finitely generated translation group $G$ of $\mathbb{R}^{3}$.

From now on we consider only algebraic surfaces. The function $g$ is the stereographic projection of the Gauß map $N: R \rightarrow S^{2}$ of the minimal immersion $X$. This minimal immersion is well defined in $\mathbb{R}^{3} / G$, but allowed to be a multivalued function in $\mathbb{R}^{3}$. The function $g$ is a covering map of $\hat{\mathbb{C}}$ and the total curvature of $\tilde{S}$ is $-4 \pi \operatorname{deg}(g)$.

\section{The Compact Riemann Surfaces $\bar{M}$ and the Functions $z$}

Denote by $M$ the surface represented in Figure 1 , and let $\mathcal{M}$ be the quotient of $M$ by its translation group. A compactification of the Scherk ends of $\mathcal{M}$ will lead to a compact Riemann surface that we call $\bar{M}$. The fundamental piece of $M$ is represented in Figure 2(a), together with some special points on it. The Scherk ends are $E_{1}$ and $E_{2}$. We have that $\bar{M}$ is invariant under reflections in the closed bold curve, indicated in Figure 2(a). The images of $S, F, E_{1}$ and $E_{2}$ under this reflection will be called $S^{\prime}, F^{\prime}, E_{1}$ and $E_{2}$, respectively.

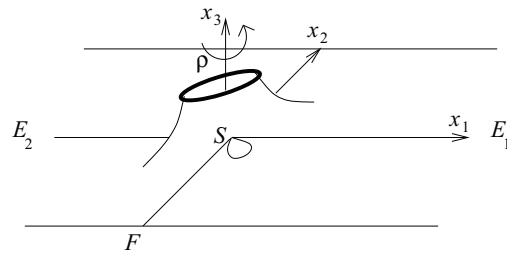

(a)

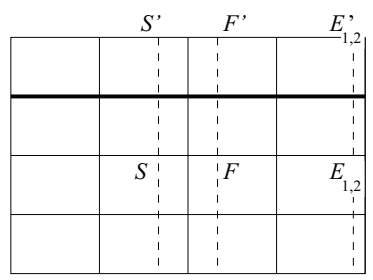

(b)

Figure 2. (a) The fundamental piece of $M$; (b) the torus $T=\rho(\bar{M})$

Let us call $\rho$ the quotient of $\bar{M}$ by the $180^{\circ}$-rotation around the axis $x_{3}$, indicated in Figure 2(a). It is easy to see that $\bar{M}$ has genus 3 and that rotation has 4 fixed points, namely $S, T, S^{\prime}, F^{\prime}$. Therefore, the Euler-Poincaré formula gives

$$
\chi(\rho(\bar{M}))=\frac{\chi(\bar{M})}{2}+\frac{4}{2}=0
$$


Because of that, $\rho(\bar{M})$ is a torus $T$. A horizontal reflectional symmetry of $\bar{M}$ is induced by $\rho$ on $T$, and since this symmetry has two components, we conclude that $T$ is a rectangular torus, represented in Figure 2(b). Now we can choose an elliptic function $Z$ on $T$, define $z:=Z \circ \rho$ and then try to deduce Weierstraß data of $M$ on $\bar{M}$ in terms of $z$. Consider Figure 3(a) and the points marked with a black square ( $\boldsymbol{\square})$ on it, which are the branch points of a certain meromorphic function $Z: T \rightarrow \mathbb{C}$ with $\operatorname{deg}(Z)=2$. Let us now take an angle $\alpha \in(0, \pi / 4)$. As indicated in Figure 3(a), we choose $Z$ such that it takes the values $\pm i \tan \alpha$ and $\pm i \cot \alpha$ at its branch points. Up to a biholomorphism, such a function is unique, and $\alpha$ determines the rectangular torus. The torus is square if and only if $\alpha=\pi / 8$.

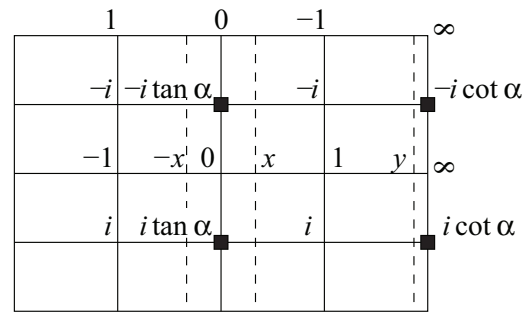

(a)

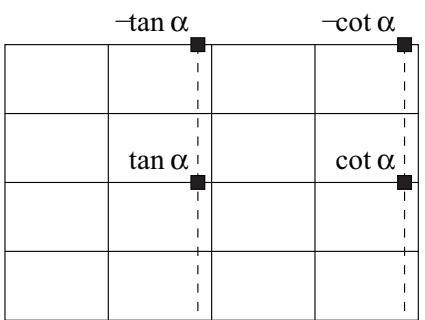

(b)

Figure 3. (a) Important values of $Z$ on $T$; (b) the function $W$ on $T$

The most important values of $Z$ are indicated in Figure 3(a). We have $Z(\rho(F))=-Z(\rho(S))=x$ for some $x \in(0, \infty)$, and $Z\left(\rho\left(E_{1,2}\right)\right)=y$, where $y^{-1} \in\left(-x^{-1}, x^{-1}\right)$. This means, we include the possibility of $y$ to be $\infty$. Consequently, $Z\left(\rho\left(S^{\prime}\right)\right)=-Z\left(\rho\left(F^{\prime}\right)\right)=x$ and $Z\left(\rho\left(E_{1,2}\right)\right)=-y$.

In the next section, we shall write the function $g$ on $\bar{M}$ in terms of $z:=Z \circ \rho$. However, this task will be simpler if we introduce another function $W: T \rightarrow \mathbb{C}$, of which the important values are presented in Figure 3(b). In fact, $W$ is a "shift" of $i Z$. We can write $W$ in terms of $Z$ and $Z$ ' by using an addition theorem for elliptic functions, or apply some easier arguments which will be explained in the next paragraph. Nevertheless, they will give us an explicit formula for $W^{2}$ instead of $W$.

Consider the following picture where $\xi$ is a pure imaginary value to be determined later:

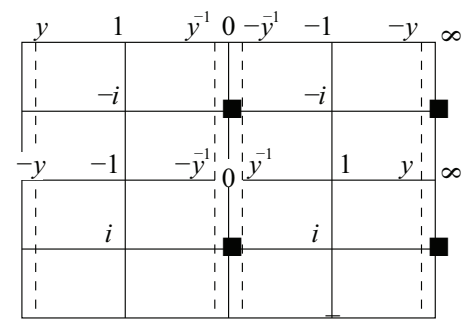

(a)

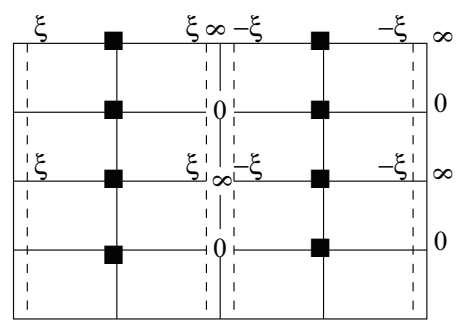

(b)

Figure 4. (a) Poles and zeros of $Z$; (b) poles and zeros of $Z^{\prime} / Z$

Let us define

$$
f:=\frac{Z^{2}-y^{-2}}{Z\left(\frac{Z^{\prime}}{Z}+\xi\right)}
$$

From Figures 3(a) and 4 it is easy to see that, for a certain complex constant $c$, the equality $c f^{2}=\frac{W^{2}-\tan ^{2} \alpha}{W^{2}-\cot ^{2} \alpha}$ holds. Based on Figure 3(a), we can easily write down an algebraic equation of $T$ as follows:

$$
Z^{\prime 2}=-\left(Z^{2}+\tan ^{2} \alpha\right)\left(Z^{2}+\cot ^{2} \alpha\right) .
$$

Therefore, $\left(\frac{Z^{\prime}}{Z}\right)^{2}=-\left(Z^{2}+Z^{-2}+\tan ^{2} \alpha+\cot ^{2} \alpha\right)$ and consequently we fix $\xi=i\left(y^{2}+y^{-2}+\tan ^{2} \alpha+\cot ^{2} \alpha\right)^{\frac{1}{2}}$. From Figure 3, it is not difficult to prove that $W=\infty$ implies $Z= \pm i\left(\frac{1+y^{2} \cot ^{2} \alpha}{y^{2}+\cot ^{2} \alpha}\right)^{\frac{1}{2}}$. Hence, 


$$
c=\left.f^{-2}\right|_{W=\infty}=\frac{\cot ^{2} \alpha \cdot y^{2}}{\left[2+\cot ^{2} \alpha \cdot\left(y^{2}+y^{-2}\right)\right]^{2}} \cdot\left(\tan ^{2} \alpha-\cot ^{2} \alpha+|\xi|^{2}\right)^{2} .
$$

The explicit relation between $W^{2}$ and $Z, Z^{\prime}$ can be given as follows

$$
W^{2}=\cot ^{2} \alpha+\frac{\cot ^{2} \alpha-\tan ^{2} \alpha}{c f^{2}-1}, \text { where } f=\frac{Z^{2}-y^{-2}}{Z^{\prime}+\xi Z} .
$$

\section{The Gauß Map of $M$ and the Function $g$ on $\bar{M}$}

Consider $z:=Z \circ \rho$ and $w:=W \circ \rho$. Therefore, $z$ and $w$ are meromorphic functions on $\bar{M}$ and $\operatorname{deg}(z)=\operatorname{deg}(w)=4$. Based on Figures 1 and 2(a), one easily sees that the unitary normal vector on $M$ is expected to be vertical at $S, F, E_{1}$ and $E_{2}$. From now on, we are going to use some heuristic arguments: if the normal vector points downwards at $S$, it will then point upwards at $F, E_{1}$ and $E_{2}$. Consequently, $g\left(\left\{S, F^{\prime}, E_{1,2}\right\}\right)=\{0\}$ and $g\left(\left\{S^{\prime}, F, E_{1,2}\right\}\right)=\{\infty\}$. We do not expect the normal vector to be vertical at any other point of $\bar{M}$, except at the ones just mentioned. Moreover, all the poles and zeros of $g$ are simple. Hence, $\operatorname{deg}(g)=4$.

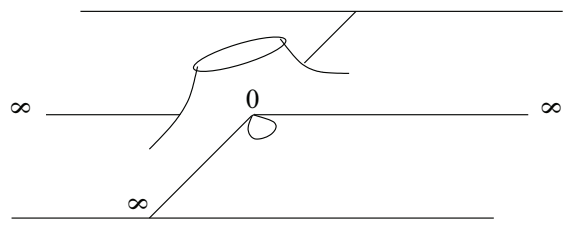

(a)

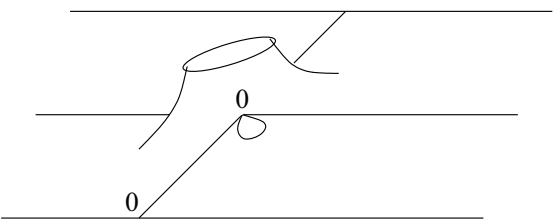

(b)

Figure 5. (a) Poles and zeros of $g$; (b) poles and zeros of $d h$

Based on Figures 3 and 5(a), one easily concludes that

$$
g^{2}=\frac{x+z}{x-z} \cdot \frac{\cot \alpha+w}{\cot \alpha-w} .
$$

Of course, a priori both sides of 3 are just proportional. However, since the unitary normal vector is expected to be horizontal on the closed bold curve in Figure 2(a), this must imply that $g$ is unitary there. Both $z$ and $w$ are pure imaginary on this curve. Therefore, the proportional constant at (3) must be unitary. Moreover, based on Figures 2 (a) and 3, the first picture suggests that $g$ is real for $z \in(-x, x)$ and pure imaginary for $z \in \mathbb{R} \backslash(-x, x)$. Since $w \in[-\cot \alpha, \cot \alpha]$ whenever $z$ is real, we conclude that the unitary proportional constant is 1 . Thus, (3) itself is already consistent with our analysis. From now on, we define $\bar{M}$ as a member of the family of compact Riemann surfaces given by (3).

By applying the Riemann-Hurwitz formula to (3), one obtains

$$
\frac{4(2-1)+8(2-1)}{2}-4+1=3 \text {. }
$$

Thus, the genus of $\bar{M}$ is three. Now we must verify that $\bar{M}$ really has all the symmetries we supposed at the beginning, and $g$ really corresponds to the unitary normal vector on $M$. First of all, let us show that $w(\bar{z})=\bar{w}(z)$ and $w(-\bar{z})=-\bar{w}(z)$. From (1) we have $Z^{\prime}(\bar{Z})= \pm \overline{Z^{\prime}}(Z)$. But according to (1), $Z^{\prime}$ is pure imaginary where $Z$ is real. Hence $Z^{\prime}(\bar{Z})=-\overline{Z^{\prime}}(Z)$. Now we recall that $\xi=i|\xi|$, use (2) and get $f(\bar{Z})=-\bar{f}(Z)$ and $W^{2}(\bar{Z})=\bar{W}^{2}(Z)$. Hence $W(\bar{Z})= \pm \bar{W}(Z)$, but since $W \in[-\cot \alpha, \cot \alpha]$ whenever $Z$ is real, then $W(\bar{Z})=\bar{W}(Z)$. Now apply $z=Z \circ \rho$ and $w=W \circ \rho$ to these relations.

By recalling (1) again, in the case $Z \rightarrow-\bar{Z}$ we do have two possibilities: either $Z^{\prime}(-\bar{Z})=\overline{Z^{\prime}}(Z)$ or $Z^{\prime}(-\bar{Z})=-\overline{Z^{\prime}}(Z)$. Nevertheless, our assumptions about the symmetries of $M$ do not imply that $\rho$ induces from $\bar{M}$ the involution $\left(Z^{\prime}, Z\right) \rightarrow\left(-\overline{Z^{\prime}},-\bar{Z}\right)$ on $T$. Hence, we only consider $\left(Z^{\prime}, Z\right) \rightarrow\left(\overline{Z^{\prime}},-\bar{Z}\right)$. From (2) it follows that $W(-\bar{Z})= \pm \bar{W}(Z)$. Since $W$ must be pure imaginary for $Z \in i \cdot[-\cot \alpha, \cot \alpha]$, then $W(-\bar{Z})=-\bar{W}(Z)$ and consequently we get $w(-\bar{z})=-\bar{w}(z)$.

Now we can summarize our study of the symmetries of $\bar{M}$ and the behaviour of $g$ in the following table: 


\begin{tabular}{ccc}
\hline involution & $z-$ values & $g$-values \\
\hline$(g, z) \rightarrow(\bar{g}, \bar{z})$ & $z=t,-x<t<x$ & $g \in \mathbb{R}$ \\
$(g, z) \rightarrow(-\bar{g}, \bar{z})$ & $z=t, x<t<y$ & $g \in i \mathbb{R}$ \\
$(g, z) \rightarrow(-\bar{g}, \bar{z})$ & $z=t, y<t<-x$ & $g \in i \mathbb{R}$ \\
$(g, z) \rightarrow(1 / \bar{g},-\bar{z})$ & $z=i t, \tan \alpha<t<\cot \alpha$ & $|g| \equiv 1$ \\
$(g, z) \rightarrow(1 / \bar{g},-\bar{z})$ & $z=i t,-\cot \alpha<t<-\tan \alpha$ & $|g| \equiv 1$ \\
\hline
\end{tabular}

\section{The Height Differential $d h$ on $\bar{M}$}

Now we are going to write down an explicit formula for $d h$, which will take into account the regular points and types of ends we want the surface $M$ to have. Based on Figures 2(a) and 3(a), one sees that $S$ and $F$ correspond to regular points of $M$, at which the normal vector is vertical. The same is valid for $S^{\prime}$ and $F^{\prime}$. Therefore, $d h\left(\left\{S, F, S^{\prime}, F^{\prime}\right\}\right)=\{0\}$. Since $M$ has only Scherk-type ends, all in the $x_{2}$-direction, then $d h$ has no poles and is holomorphic on $\bar{M}$. Moreover, since $\operatorname{deg}(d h)=4$, we conclude that all zeros of $d h$ are simple. They are represented in Figure 5(b).

For convenience of the reader, we reproduce here the algebraic equation of $T$ already established in (1):

$$
Z^{\prime 2}=-\left(Z^{2}+\tan ^{2} \alpha\right)\left(Z^{2}+\cot ^{2} \alpha\right) .
$$

From Figure 5(b) one immediately verifies that

$$
d h=\frac{d z}{Z^{\prime} \circ \rho} .
$$

A priori, both sides of (6) are just proportional, but since $z$ is real on the straight lines of $\bar{M}$, from (5) we get pure imaginary values for $Z^{\prime} \circ \rho$ on these lines. Therefore, the proportional constant at (6) must be real, and we choose it to be 1 . This will imply that (6) is also consistent with $\operatorname{Re}\{d h\}=0$ on the closed bold curve in Figure 2(a). There we have $z=i t, \tan \alpha<|t|<\cot \alpha$, which leads to real values for $Z^{\prime} \circ \rho$.

Analogously, we could also have defined the algebraic equation of $T$ by $W^{\prime 2}=\left(W^{2}-\tan ^{2} \alpha\right)\left(W^{2}-\cot ^{2} \alpha\right)$ and so $d h=d w / W^{\prime} \circ \rho$, namely

$$
d h=\frac{d w}{\sqrt{\left(w^{2}-\tan ^{2} \alpha\right)\left(w^{2}-\cot ^{2} \alpha\right)}} .
$$

Exactly at this point, we need to prove that $M$ really has the planar geodesics and straight lines of our initial assumption. This task is summarized in the following table:

\begin{tabular}{ccc}
\hline$z=t,-x<t<x$ & $g \in \mathbb{R}$ & $d h(\dot{z}) \in i \mathbb{R}$ \\
$z=t, x<t<y$ & $g \in i \mathbb{R}$ & $d h(\dot{z}) \in i \mathbb{R}$ \\
$z=t, y<t<-x$ & $g \in i \mathbb{R}$ & $d h(\dot{z}) \in i \mathbb{R}$ \\
$z=i t, \tan \alpha<t<\cot \alpha$ & $|g| \equiv 1$ & $d h(\dot{z}) \in i \mathbb{R}$ \\
$z=i t,-\cot \alpha<t<-\tan \alpha$ & $|g| \equiv 1$ & $d h(\dot{z}) \in i \mathbb{R}$ \\
\hline
\end{tabular}

From this table, it is immediate to verify that $d h \cdot d g / g$ is real on the expected planar geodesics of $M$, and pure imaginary on the expected straight lines of $M$. We now have the Weierstraß data $(g, d h)$ on $T$, given by (3) and (7), and the free parameters $\alpha, x$ and $y$.

\section{Solution of the Period Problems}

Let us consider Figure 6. It reproduces Figure 2(a) and its image under $z$ with some special paths indicated there.

Around the punctures of $\bar{M}$, namely $w^{-1}(\{ \pm \cot \alpha\})$, we consider small curves given by $w(t)= \pm \cot \alpha+\delta e^{i t}$, where $\delta$ is a positive real and $t$ varies in the interval $[0,4 \pi]$ (we recall that $w$ takes the values $\pm \cot \alpha$ with multiplicity 2). All these curves are homotopically equivalent for sufficiently small values of $\delta$. Therefore, by letting $\delta \rightarrow 0$ an immediate calculation leads to $\operatorname{Re} \oint\left(\phi_{1}, \phi_{3}\right)=0$, and up to a minus sign, $\operatorname{Re} \oint \phi_{2}=\left.\operatorname{Res}\left(\phi_{2}\right)\right|_{w=\cot \alpha}$, where

$$
\left.\operatorname{Res}\left(\phi_{2}\right)\right|_{w=\cot \alpha}=\frac{2 \pi}{\sqrt{\cot ^{2} \alpha-\tan ^{2} \alpha}} \cdot \sqrt{\frac{y+x}{y-x}} .
$$




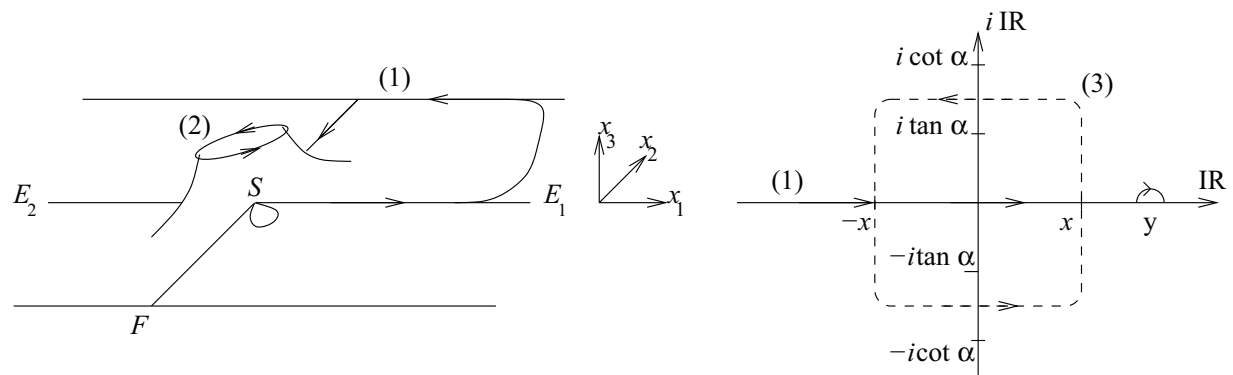

Figure 6. The fundamental piece of $M$ and its image under $z$

Based on this analysis and (7), it is clear that $\operatorname{Re} \int_{(1)} \phi_{3}=0$. Moreover, (7) also gives us $\operatorname{Re} \int_{(2)} \phi_{3}=0$. The curve (2) from Figure 6 is homotopically equivalent to the sum of (1) with its image under the maps $(g, z) \rightarrow(\bar{g}, \bar{z})$ and $(g, z) \rightarrow(-\bar{g}, \bar{z})$, composed in this order (see Table 4). Actually, this composition corresponds to the rotation $\rho$, explained at the beginning of Section 3. Since $\int_{\rho \circ(1)}\left(\phi_{1}, \phi_{2}\right)=-\int_{(1)}\left(\phi_{1}, \phi_{2}\right)$, then $\operatorname{Re} \int_{(2)}\left(\phi_{1}, \phi_{2}\right)=0$. It remains to prove that

$$
\operatorname{Re} \int_{(1)}\left(\phi_{1}, \phi_{2}\right)=0 \text {. }
$$

In Figure 6, the curve (3) is symmetric with respect to the geodesic (2). Because of that, the only non-zero component of the period vector $\operatorname{Re} \int_{(3)} \phi_{1,2,3}$ must be the third one. Moreover, $\operatorname{Re} \int_{(3)} \phi_{3} \neq 0$ because (7) implies that $d h$ is real and never vanishes on the dashed lines of Figure 3(b). In fact, this component provides the vertical period of $M$, suggested by Figure 1. The horizontal period is given by (8).

We have just reduced the period problems to the proof of (9). For this purpose, we shall apply the limit-method cited at the introduction. Let us show that the Weierstraß data (3) and (6) converge to the Weierstraß data from Callahan-Hoffman-Meeks's surface of genus 3 (see Figure 7).

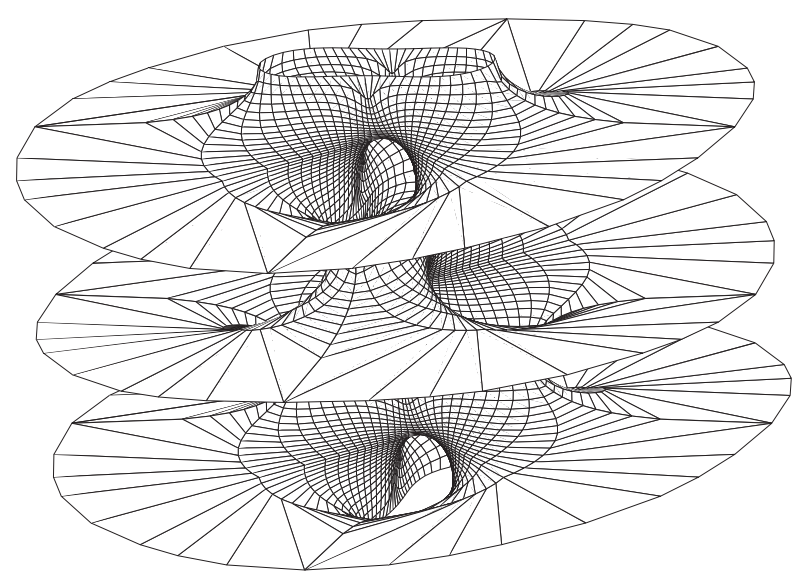

Figure 7. The Callahan-Hoffman-Meeks's surface of genus 3

Consider $K$ a compact subset of $T \backslash w^{-1}(\{ \pm \cot \alpha\})$. From Figure 8 one sees that $\left(\frac{x+z}{x-z}\right)^{2}$ converges uniformly in $K$ to $\left(\frac{w-\tan \alpha}{w+\tan \alpha}\right)\left(\frac{\cot \alpha+w}{\cot \alpha-w}\right)$ when both $x$ and $y$ approach 1. Thus, from (3) it follows that

$$
g^{4}=\left(\frac{w-\tan \alpha}{\tan \alpha+w}\right)\left(\frac{\cot \alpha+w}{\cot \alpha-w}\right)^{3} .
$$

By comparing (7) and (10) with Callahan, Hoffman, and Meeks (1989, p. 502), one sees that the Weierstraß data of our surfaces coincide in the limit, inside the compact $K$. The uniform convergence, applied to Theorem 2 , guarantees that the three coordinate functions of the minimal immersion $\left.X\right|_{K}$ will give as limit the CallahanHoffman-Meeks' example of genus 3. Of course, the convergence is restricted to $K$, but one may take this compact 
as $T$ minus arbitrarily small neighbourhoods of the two points $w^{-1}(\{ \pm \cot \alpha\})$. Therefore, we may consider that the curve (1) is entirely in $K$.

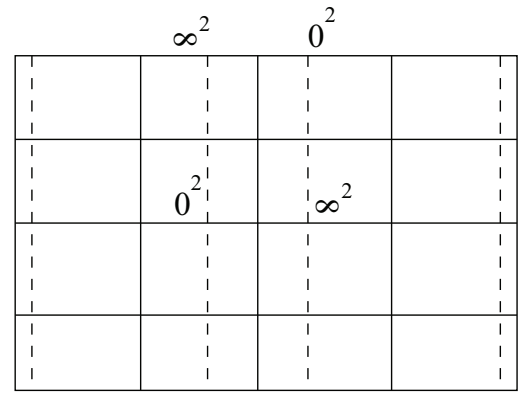

(a)

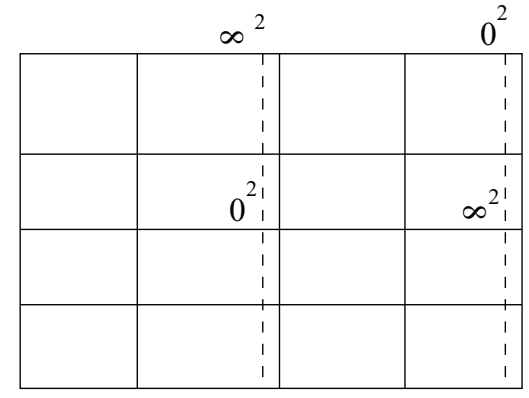

(b)

Figure 8. (a) Divisor of $\left(\frac{x+z}{x-z}\right)^{2}$; (b) divisor of $\left(\frac{w-\tan \alpha}{w+\tan \alpha}\right)\left(\frac{\cot \alpha+w}{\cot \alpha-w}\right)$

This means, although the limit passes from doubly to singly periodic surfaces, and from Scherk to planar ends, we shall not have to worry about it. Our analysis can be restricted to $\phi_{1,2}$ on the curve (1) inside $K$. This is the first step to solve (9).

Remark 1 If $\phi_{1,2,3}$ are the Weierstraß data for $(g, d h)$, we call $\widetilde{\phi}_{1,2,3}$ the ones for $(\widetilde{g}, d h):=\left(e^{-i \pi / 4} g, d h\right)$. In the case of Callahan, Hoffman, and Meeks (1989), one automatically has $\operatorname{Re} \int_{(1)} \widetilde{\phi}_{1}=0$ due to the additional symmetries.

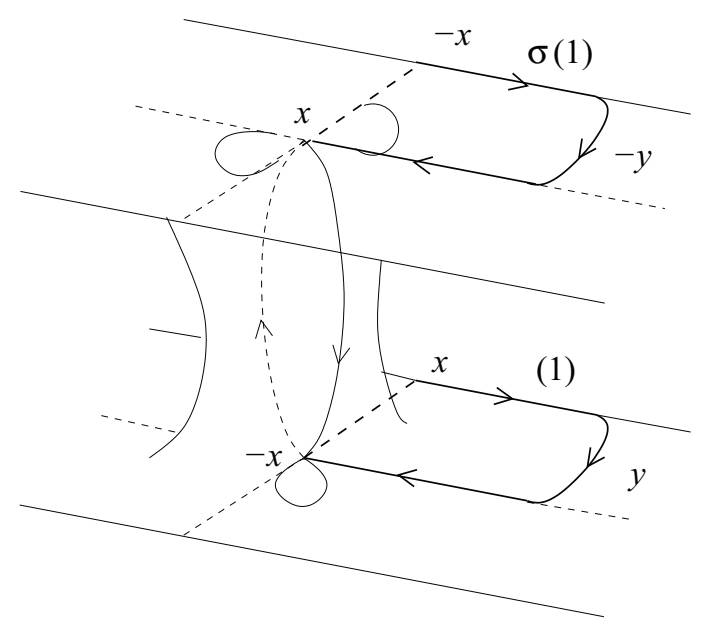

Figure 9. Description of (1) and its image under $\sigma$

Let $\sigma$ be the involution given by $(g, z) \rightarrow(-1 / \bar{g},-\bar{z})$. From (7) and recalling that $w(-\bar{z})=-\bar{w}(z)$ we have $d h \rightarrow$ $-\overline{d h}$. Therefore

$$
\operatorname{Re} \int_{\sigma(1)} \frac{d h}{g}=\operatorname{Re} \int_{(1)} \sigma^{*}\left(\frac{d h}{g}\right)=\operatorname{Re} \int_{(1)} \overline{g d h} .
$$

Figure 9 suggests that (1) is homotopic to the concatenation of $\beta$ with $\sigma(1)$, where $\beta$ represents the vertical loop and $\sigma(1)$ comes from the involution $\sigma$ applied to (1). This fact can be verified in the complex plane. Hence

$$
\begin{aligned}
\operatorname{Re} \int_{(1)} \phi_{1} & =\operatorname{Re} \int_{(1)} \frac{d h}{g}-\operatorname{Re} \int_{(1)} g d h \\
& =\operatorname{Re} \int_{(1)} \frac{d h}{g}-\operatorname{Re} \int_{\beta \cup \sigma(1)} g d h \\
& =\operatorname{Re} \int_{(1)} \frac{d h}{g}-\operatorname{Re} \int_{\sigma(1)} g d h-\operatorname{Re} \int_{\beta} g d h \\
& =-\operatorname{Re} \int_{\beta} g d h, \text { since (1) is a real curve. }
\end{aligned}
$$


We split the vertical loop as $\beta:=\beta^{+} \cup \beta^{-}$, where $\beta^{+}$is the ascending curve from $-x$ to $x$ and $\beta^{-}$the path from $x$ to $-x$. The rotation $\rho$, introduced in Section 3, corresponds to $(g, z) \rightarrow(-g, z)$, whence $g \rightarrow-g$ and $d h \rightarrow d h$ under its action. Therefore,

$$
\int_{\beta} g d h=\int_{\beta^{+} \cup \beta^{-}} g d h=2 \int_{\beta^{+}} g d h=2 \int_{\beta^{-}} g d h .
$$

Remark 2 Figure 10 represents the image under $g$ of a fundamental domain $\mathcal{D}$, namely a smallest subset of $X(M)$ that fully generates it by isometries of $\mathbb{R}^{3}$. The left image corresponds to $\left.g\right|_{\mathcal{D}}$ referring to the "front piece", which contains $\beta^{-}$. The right image concerns the "back piece", which contains $\beta^{+}$.
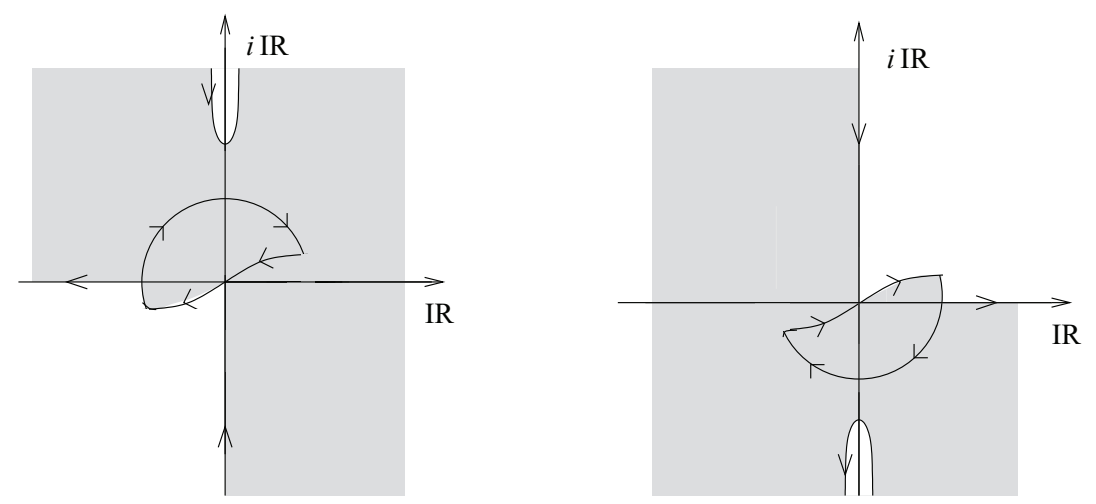

Figure 10. The image under $g$ of a fundamental domain

Before going ahead, notice that we had settled $|y|>|x|$ in Section 3. By taking $0<y<x$, one simply changes the direction of the Scherk-ends, while $x=y$ gives a planar end (see Figure 11).

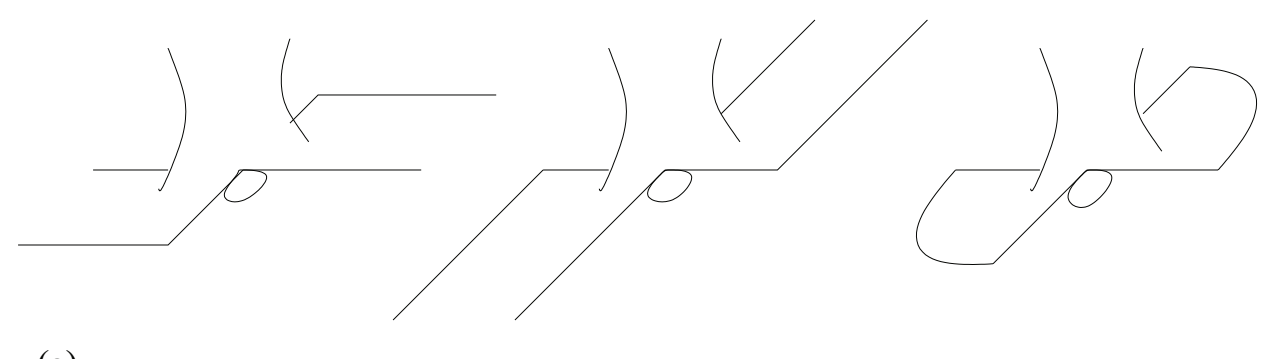

(a)

(b)

(c)

Figure 11. (a) $x<y$, (b) $x>y$, (c) $x=y$

These other choices will now be useful to close the periods. Hence, we consider the following cases:

Case I $x=1 \lesssim y$.

For $\beta^{+}$we have $z(t)=-e^{i t}$ with $0 \leq t \leq \pi$. Hence $d h$ is given by

$$
d h=\frac{-i d z / z}{\left[z^{2}+z^{-2}+\tan ^{2} \alpha+\cot ^{2} \alpha\right]^{1 / 2}}=\frac{d t}{\left[2 \cos 2 t+\tan ^{2} \alpha+\cot ^{2} \alpha\right]^{1 / 2}},
$$

and $\tan \alpha>w(t)>-\tan \alpha$. Moreover, $\left.\frac{1+z}{1-z}\right|_{\beta^{+}}=\frac{-i \sin t}{1+\cos t}$, whence $\frac{\cot \alpha+w}{\cot \alpha-w}$ and consequently $g^{2}(t)$ vary according to Figure 12.

From Remarks 1 and 2, we notice that the curve in Figure 12(b), rotated by $-\pi / 2$, has a branch of square root indicated in Figure 13(a). Therefore $\operatorname{Re} \int_{(1)} \widetilde{g} d h<0$. 


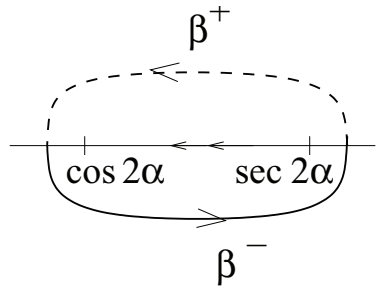

(a)

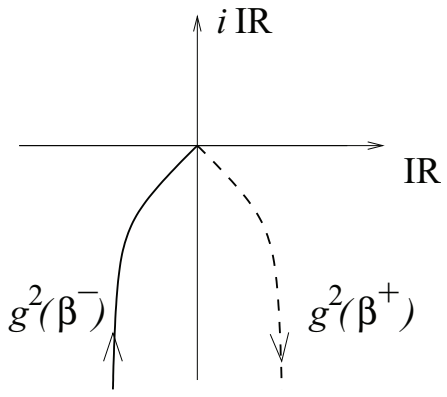

(b)

Figure 12. (a) Image of $\frac{\cot \alpha+w}{\cot \alpha-w}$; (b) image of $g^{2}$

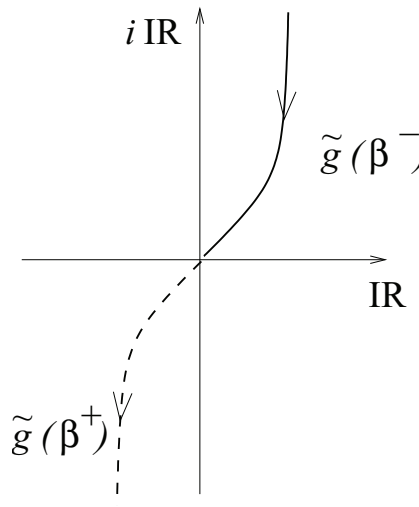

(a)

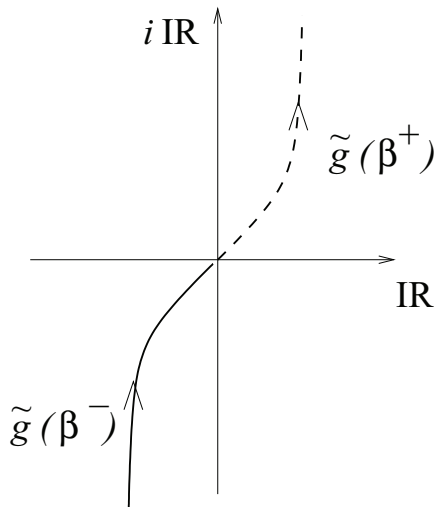

(b)

Figure 13. (a) Branch of square root of $\widetilde{g}$ in Case I; (b) in Case II

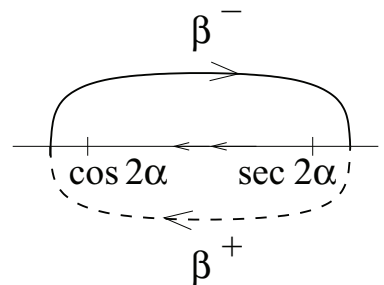

(a)

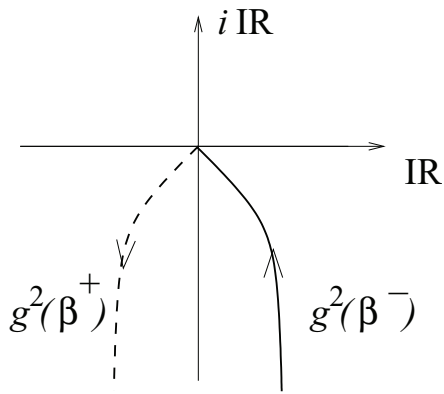

(b)

Figure 14. (a) Image of $\beta$ in Case II; (b) image of $g^{2}$

Case II $x=1 \gtrsim y$.

Now $\beta^{+}$is still given by $z(t)=-e^{i t}$ with $0 \leq t \leq \pi$ and $\frac{1+z}{1-z}=\frac{-i \sin t}{1+\cos t}$. However, since $x \gtrsim y$ we have that $\frac{\cot \alpha+w}{\cot \alpha-w}$ and consequently $g^{2}(t)$ vary according to Figure 14 . Again from Remarks 1 and 2, the branch of square root for $\widetilde{g}^{2}(t)$ is now indicated in Figure 13(b). Thus $\operatorname{Re} \int_{(1)} \widetilde{g} d h>0$.

Therefore, $\operatorname{Re} \int_{(1)} \widetilde{g} d h=0$ for some values of $(x, y)$ in a neighbourhood of $(1,1)$. At this limit-point, the function $\operatorname{Re} \int_{(1)} \widetilde{\phi}_{2}$ depends only on the parameter $\alpha \in(0, \pi / 4)$. This is the one-dimensional period problem for Callahan, Hoffman and Meeks (1989). According to Martín and Rodríguez (1997), it has only one zero that we call $\alpha^{*}$.

We can illustrate this fact by taking a vertical axis $v$ and plotting a graph on the plane $O \alpha v$, which crosses the horizontal axis at $\alpha^{*}$. Back to our surfaces, if the extra parameters $(x, y)$ were restricted to a curve $(x(\kappa), y(\kappa))$ with an extreme at $(1,1)$, then we could visualize $\kappa$ as a third axis to $O \alpha v$. In this way, both $\operatorname{Re} \int_{(1)} \widetilde{\phi}_{1,2}$ turn out to be dependent on two variables, namely $(\alpha, \kappa)$, and their graphs are surfaces like Figure 15 suggests. Notice 
that we cannot provide numeric pictures of this fact, since our analyses include limit-values. They typically make unreliable any computational image. This is the second step to solve (9).

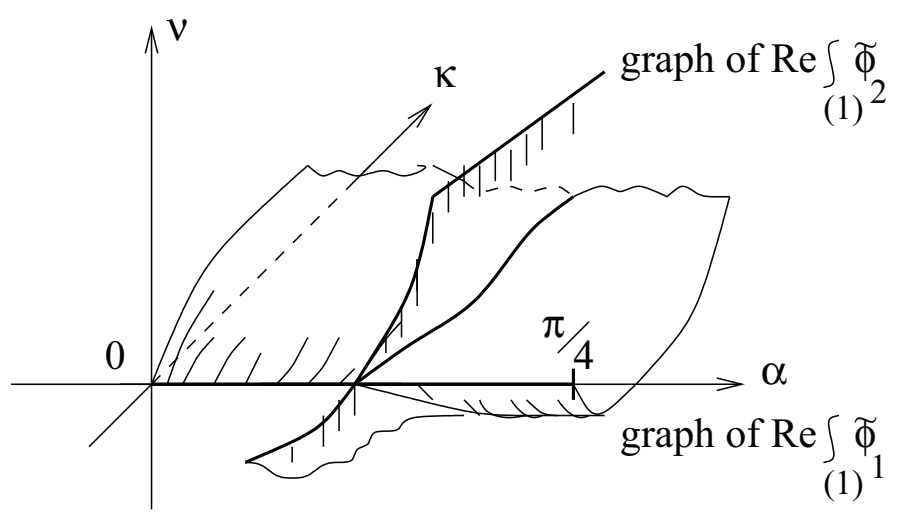

Figure 15. Periods on curve (1)

Let us take, for instance, $x$ and $y$ as functions of $(\alpha, \kappa)$ given by $x=1+\kappa \alpha, y=1+\kappa \alpha+2 \kappa\left(\frac{4 \alpha^{*}+\pi}{8}-\alpha\right)$. Hence $\kappa \gtrsim 0$ implies $x=1<y$ for $\alpha \in\left(0, \frac{4 \alpha^{*}+\pi}{8}-\alpha\right)$ and $x=1>y$ for $\alpha \in\left(\frac{4 \alpha^{*}+\pi}{8}-\alpha, \frac{\pi}{4}\right)$. Namely, $\operatorname{Re} \int_{(1)}^{8} \widetilde{\phi}_{1}<0$ in the first interval and $\operatorname{Re} \int_{(1)} \widetilde{\phi}_{1}>0$ in the second. We could extend $y(\alpha, \kappa)$ to $y=1+\kappa \alpha+2 \kappa\left(\frac{4 \alpha^{*}+\pi}{8} \cdot s-\alpha\right), 1 \geq s>0$. Consequently, there exists a curve $(\alpha(t), \kappa(t))$ for which $\operatorname{Re} \int_{(1)} \widetilde{\phi}_{1,2}=0$, where $t \in(0, \varepsilon)$ for a certain small $\varepsilon>0$ and $\lim _{t \rightarrow 0}(\alpha(t), K(t))=\left(\alpha^{*}, 0\right)$. Moreover, along this curve we have $x \neq y$ as explained next.

If $x=y \neq 1$, we assert that the period $\operatorname{Re} \int_{(1)} \widetilde{\phi}_{1}$ is non-zero in the $x_{1}$-direction. This is because one gets a CHMsurface with "torsion", as illustrated in Figure 16(b). Without torsion, on $\beta$ one has real $d h$ and $\widetilde{g}=-i|\vec{g}|$, whence $\operatorname{Re} \int_{\beta} \widetilde{g} d h=0$. For $x=y \neq 1$, however, we may still set $\left.d h\right|_{\beta^{+}}$to be real and positive, but then $\left.\widetilde{g}\right|_{\beta^{+}}$gets a nevervanishing real part. Therefore, from (11) and (12) one has $\operatorname{Re} \int_{(1)} \widetilde{\phi}_{1} d h \neq 0$. This third step finally proves (9) and concludes the present section.
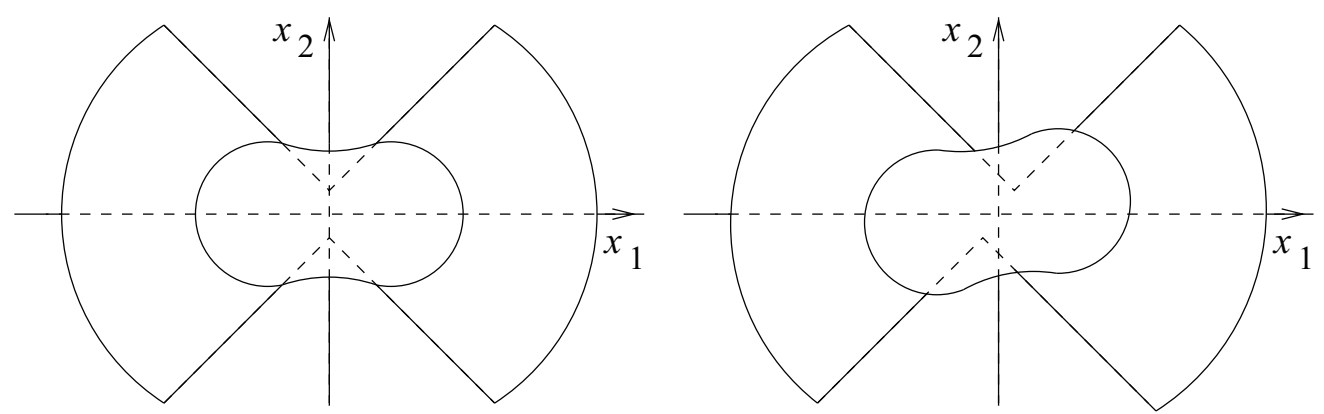

Figure 16. Symmetries for $x=y=1$ (CHM) and $x=y \neq 1$ (CHM with torsion)

\section{Embeddedness of the Fundamental Piece}

This chapter is strongly based on the ideas of Martín and Ramos Batista (2006) and Ramos Batista (2005). We begin with by identifying a fundamental domain $\mathcal{D}$ of $X(M)$ in Figure 17.

In the previous section we proved the existence of a curve $(x(t), y(t)), 0 \leq t<1$, along which (9) holds. Moreover, $\lim _{t \rightarrow 0} \alpha(t)=\alpha^{*}$. Let us fix $t \in(0,1)$ and consider the minimal immersion $X_{t}: \mathcal{D} \backslash\left\{E_{1,2}\right\} \rightarrow \mathbb{R}^{3}$, defined by (3) and (6). Consider Table (4) and take the composite of the first two involutions described there. We get $(g, z) \rightarrow(-g, z)$, and from the Weierstraß formula in Theorem 2, one easily sees that it leads to a $180^{\circ}$-rotation about the vertical axis, providing one takes $X_{t}(S)$ as the origin. This means, any $q \in \mathcal{D} \backslash\left\{E_{1,2}\right\}$ is taken to a pair of points in $\mathbb{R}^{3}$, say $X_{t}(q)^{+}$and $X_{t}(q)^{-}$, and one is the image of the other by a $180^{\circ}$ rotation around $O x_{3}$. 
We now describe the fundamental piece $P$ of $M$. Let $P^{-}$be the image of $\mathcal{D} \backslash\left\{E_{1,2}\right\}$ in $\mathbb{R}^{3}$ under $X_{t}$, and $P^{+}$the image of $P^{-}$in $\mathbb{R}^{3}$ under a $180^{\circ}$ rotation around $X_{t}([-x, x])$. Thus $P=P^{+} \cup P^{-}$.

Let $V_{E}$ be a connected, arbitrarily small neighbourhood of the point $E_{1,2}$. In Section 6, we saw that (7) and (10) provided a uniform convergence of $(g, d h)$ to the Weierstraß data of the embedded CHM-surface. Let us denote this minimal embedding by $X_{0}$.

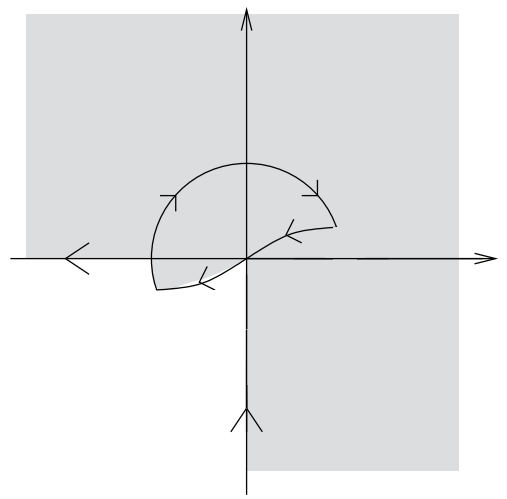

(a)

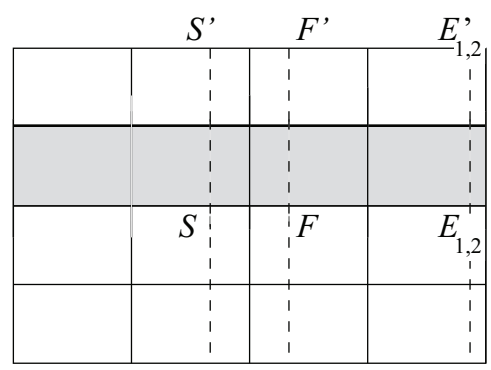

(b)

Figure 17. (a) The set $g(\mathcal{D})$; (b) the image of the fundamental domain $\mathcal{D}$ under $\rho$

Define $\mathcal{K}=\mathcal{D} \backslash V_{E}$, which is a compact set. Since $X_{t}$ converges to CHM in $\mathcal{K}$, then we could have chosen $V_{E}$ so small that $\left.X_{0}\right|_{\partial V_{E}}$ would consist of two space curves with injective projections $\Gamma^{ \pm}$in the plane $x_{3}=0$ (see Figure 18). This is because a planar end is a graph, as proved in Schoen (1983). By the way, because of that we may consider that $\Gamma^{ \pm}$consists of four half-lines, whose extension is $O x_{1} \cup O x_{2}$, joined at their origins by two $90^{\circ}$-arcs of circumference. Moreover, the smaller $V_{E}$ gets, the more $g\left(V_{E}\right)$ shrinks as a set containing $\infty \in \hat{\mathbb{C}}$. In particular, $g\left(V_{E}\right)$ turns out to be a subset of the upper hemisphere.

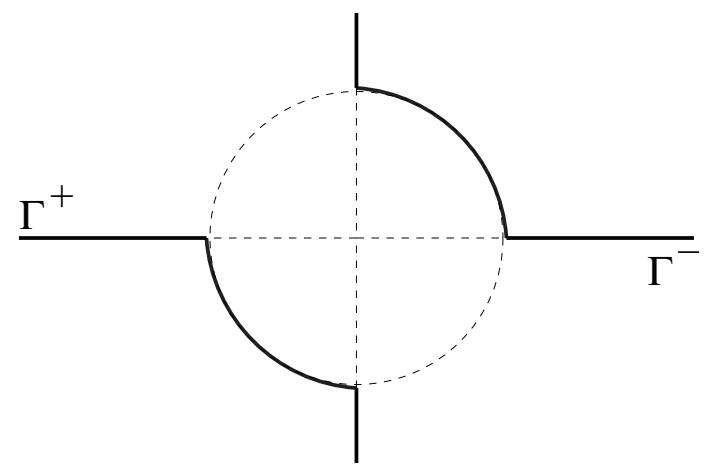

Figure 18. The curves $\Gamma^{ \pm}$

Since the convergence $X_{t} \rightarrow X_{0}$ is uniform on compact sets, for $t$ close enough to zero the projection of $\left.X_{t}\right|_{\partial V_{E}}$ onto $x_{3}=0$ is close to $\Gamma^{ \pm}$. However, it now consists of two curves $C^{ \pm}$which determine two simply connected open regions $R^{+}$and $R^{-}$(see Figure 19).

We recall that $g\left(V_{E}\right)$ is contained in the upper hemisphere. Therefore, $\left.\left(x_{1}, x_{2}\right)\right|_{V_{E}}$ is an immersion whose image has $C^{ \pm}$as boundary. Since $x_{2}$ is bounded for any fixed $t \in(0,1)$, then $\left.\left(x_{1}, x_{2}\right)\right|_{V_{E}}=R^{+} \cup R^{-}$. Since $\partial R^{ \pm}$are the monotone curves $C^{ \pm}$, then $\left.X_{T}\right|_{\partial V_{E}}$ is a graph of $x_{3}$ as a function of $\left(x_{1}, x_{2}\right)$.

Now observe that $\left.X_{0}\right|_{\mathcal{K}}$ is a compact embedded minimal surface in $\mathbb{R}^{3}$. Since its boundary does not have selfintersections, then $\left.X_{t}\right|_{\mathcal{K}}$ is still embedded for sufficiently small $t$. Moreover, $\left.X_{t}\right|_{\mathcal{K}}$ does not intercept $\left.X_{t}\right|_{V_{E}}$, otherwise there would be a ball in $\mathbb{R}^{3}$ containing the whole boundary of $\left.X_{t}\right|_{\mathcal{K}}$ but not all the rest of it. This is impossible according to the maximum principle. Hence, the pieces $X_{t} \mid \mathcal{K}$ and $\left.X_{t}\right|_{V_{E}}$ make together a minimal embedding $X_{t}$ : $\mathcal{D} \backslash\left\{E_{1,2}\right\} \rightarrow \mathbb{R}^{3}$, for $t$ sufficiently close to zero. 
Again by the maximum principle, we may extend this conclusion for all $t \in(0,1)$. Therefore, $P^{+}$is embedded in $\mathbb{R}^{3}$, and since $P^{-}$is its image under a $180^{\circ}$ rotation about the segment of $P^{+}$, the whole piece $P$ will not have self-intersections. Since the immersion is proper, then $P$ is embedded in $\mathbb{R}^{3}$.

Now $P \subset \mathbb{R}^{3} / \mathcal{G}$, where $\mathcal{G}$ is the group of $\mathbb{R}^{3}$ generated by $\left(x_{1}, x_{2}, x_{3}\right) \rightarrow\left(x_{1}, x_{2},-x_{3}+2 \operatorname{Re} \int_{\beta^{+}} d h\right)$ and $\left(x_{1}, x_{2}, x_{3}\right) \rightarrow$ $\left(x_{1}, x_{2}+\operatorname{Re} \oint \phi_{2}, x_{3}\right)$. In the horizontal faces of $\partial\left(\mathbb{R}^{3} / \mathcal{G}\right)$ we have the reflection curves of $P$. In the vertical faces we have the straight lines of $P$. By applying $\mathcal{G}$ to $P$ one generates $M$, which is then complete, doubly periodic and embedded in $\mathbb{R}^{3}$.

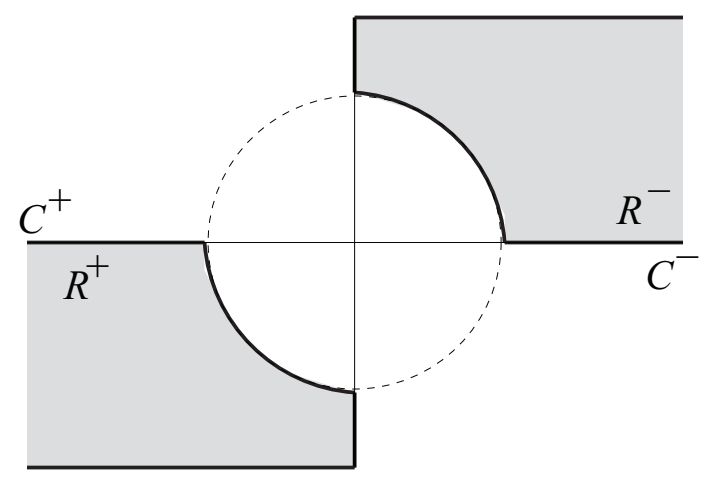

Figure 19. Regions $R^{ \pm}$and curves $C^{ \pm}$

\section{References}

Baginski, F., \& Ramos Batista, V. (2011). Solving period problems for minimal surfaces with the support function. Adv. Appl. Math. Sci., 9, 85-114.

Callahan, M., Hoffman, D., \& Meeks, W. (1989). Embedded minimal surfaces with an infinite number of ends. Invent. Math., 96, 459-505. http://dx.doi.org/10.1007/BF01393694

Hart, G. (2007). Where are nature's missing structures? Nature Materials, 6, 941-945. http://dx.doi.org/10.1038/nmat2057

Hauswirth, L., Morabito, F., \& Rodíguez, M. (2009). An end-to-end-construction for singly periodic minimal surfaces. Pacific J. Math., 241, 1-61. http://dx.doi.org/10.2140/pjm.2009.241.1

Hoffman, D., Karcher, H., \& Wei, F. (1993). The genus one helicoid and the minimal surfaces that led to its discovery. Global Analysis and Modern Mathematics, Publish or Perish Press, 119-170.

Hoffman, D., Wolf, M., \& Weber, M. (2009). An embedded genus-one helicoid. Ann. Math., 169, 347-448. http://dx.doi.org/10.4007/annals.2009.169.347

Jenkins, H., \& Serrin, J. (1966). Variational problems of minimal surface type. II. Boundary value problems for the minimal surface equation. Arch. Rational Mech. Anal., 21, 321-342. http://dx.doi.org/10.1007/BF00282252

Kapouleas, N. (1997). Complete embedded minimal surfaces of finite total curvature. J. Differential Geom., 47, 95-169.

Karcher, H. (1988). Embedded minimal surfaces derived from Scherk's examples. Manuscr. Math., 62, 83-114. http://dx.doi.org/10.1007/BF01258269

Karcher, H. (1989). Construction of minimal surfaces. Lecture Notes 12, SFB256, Bonn.

Karcher, K., \& Polthier, K. (1996). Construction of triply periodic minimal surfaces. Differentialgeometrie und Quantenphysik, SFB288 194, Berlin.

López, F., \& Martín, F. (1999). Complete minimal surfaces in $\mathbb{R}^{3}$. Publ. Mat., 43, 341-449. http://dx.doi.org/10.5565/PUBLMAT_43299_01

Lord, E., \& Mackay, A. (2003). Periodic minimal surfaces of cubic symmetry. Current Science, 85, 346-362.

Lübeck, K. (2007). Método-limite para solução de problemas de períodos em superfícies mínimas. Doctoral Thesis, Campinas. 
Lübeck, K., \& Ramos Batista, V. (2009). A limit-method for solving period problems on minimal surfaces. Math. Z., 263, 633-646. http://dx.doi.org/10.1007/s00209-008-0435-1

Martín, F., \& Ramos Batista, V. (2006). The embedded singly periodic Scherk-Costa surfaces. Math. Ann., 336, 155-189. http://dx.doi.org/10.1007/s00208-006-0778-z

Martín, F., \& Rodríguez, D. (1997). A characterization of the periodic Callahan-Hoffman-Meeks surfaces in terms of their symmetries. Duke Math. J., 89, 445-463.

Meeks, W., \& Rosenberg, H. (1989). The global theory of doubly periodic minimal surfaces. Invent. Math., 97, 351-379. http://dx.doi.org/10.1007/BF01389046

Meeks, W., \& Rosenberg, H. (2005). The uniqueness of the helicoid. Ann. Math., 161, 727-758. http://dx.doi.org/10.4007/annals.2005.161.727

Nitsche, J. (1989). Lectures on minimal surfaces. Cambridge: Cambridge University Press.

Osserman, R. (1986). A survey of minimal surfaces (2nd ed.). New York: Dover.

Pérez, J., Rodríguez, M., \& Traizet, M. (2005). The classification of doubly periodic minimal tori with parallel ends. J. Differential Geom., 69, 523-577.

Ramos Batista, V. (2000). Construction of new complete minimal surfaces in $\mathbb{R}^{3}$ based on the Costa surface. Doctoral Thesis, University of Bonn, Bonn.

Ramos Batista, V. (2003). A family of triply periodic Costa surfaces. Pacific J. Math., 212, $347-370$. http://dx.doi.org/10.2140/pjm.2003.212.347

Ramos Batista, V. (2004). Noncongruent minimal surfaces with the same symmetries and conformal structure. Tohoku Math. J., 56, 237-254.

Ramos Batista, V. (2005). Singly periodic Costa surfaces. J. London Math. Soc., 72, $478-496$. http://dx.doi.org/10.1112/S0024610705006836

Rossman, W., Thayer, E., \& Wohlgemuth, M. (2000). Embedded, doubly periodic minimal surfaces. Experiment. Math., 9, 197-219. http://dx.doi.org/10.1080/10586458.2000.10504646

Schoen, R. (1983). Uniqueness, symmetry and embeddedness of minimal surfaces. J. Differential Geom., 18, 701-809.

Traizet, M. (1996). Construction de surfaces minimales en recollant des surfaces de Scherk. Ann. Inst. Fourier, 46, 1385-1442. http://dx.doi.org/10.5802/aif.1554

Traizet, M. (2002a). An embedded minimal surface with no symmetries. J. Differential Geom., 60, 103-153.

Traizet, M. (2002b). Adding handles to Riemann's minimal surfaces. J. Inst. Math. Jussieu, 1, $145-174$. http://dx.doi.org/10.1017/S147474800200004X

Weber, M. (2000). A Teichmüller theoretical construction of high genus singly periodic minimal surfaces invariant under a translation. Manuscripta Math., 101, 125-142.

Wei, F. (1992). Some existence and uniqueness theorems for doubly periodic minimal surfaces. Invent. Math., 109, 113-136. http://dx.doi.org/10.1007/BF01232021

\section{Copyrights}

Copyright for this article is retained by the author(s), with first publication rights granted to the journal.

This is an open-access article distributed under the terms and conditions of the Creative Commons Attribution license (http://creativecommons.org/licenses/by/3.0/). 